University of Wollongong

Research Online

Australian Institute for Innovative Materials -

Papers

Australian Institute for Innovative Materials

$1-1-2019$

Hierarchically stacked reduced graphene oxide/carbon nanotubes for as high performance anode for sodium-ion batteries

Jianmin Feng

Tianjin Normal University

Lei Dong

Tianjin Normal University

Xifei Li

Tianjin Normal University

Dejun Li

Tianjin Normal University

Pengyi Lu

Tianjin University

See next page for additional authors

Follow this and additional works at: https://ro.uow.edu.au/aiimpapers

Part of the Engineering Commons, and the Physical Sciences and Mathematics Commons

Research Online is the open access institutional repository for the University of Wollongong. For further information contact the UOW Library: research-pubs@uow.edu.au 


\title{
Hierarchically stacked reduced graphene oxide/carbon nanotubes for as high performance anode for sodium-ion batteries
}

\author{
Abstract \\ Sodium has attracted an increasing amount of attention as an alternative element to lithium for energy \\ storage due to its low cost and wide distribution, although the intercalation problem for sodium ions in \\ conventional anode materials, due to their larger ionic size, still has to be overcome in order to utilize this \\ element. Herein, we report a carbon-based hybrid material that is composed of stacked reduced graphene \\ oxide/carbon nanotubes (rGO/CNTs) with a hierarchical and open structure to accommodate $\mathrm{Na}$ ions, \\ which was fabricated by a liquid-phase oxidative exfoliation and subsequent reduction. This rGO/CNTs \\ hybrid material features a hierarchical nanostructure with rGO nanosheets homogeneously spaced by \\ monodisperse CNTs. The increased interlayer space between individual rGO nanosheets, which resulted \\ from the inserted CNTs, is beneficial for highly efficient and reversible $\mathrm{Na}$ ion intercalation, leading to a \\ high and stable capacity of $295 \mathrm{mAhg}-1$ at $50 \mathrm{mAg}-1$ for 200 cycles. \\ Disciplines \\ Engineering | Physical Sciences and Mathematics

\section{Publication Details} \\ Feng, J., Dong, L., Li, X., Li, D., Lu, P., Hou, F., Liang, J. \& Dou, S. Xue. (2019). Hierarchically stacked reduced \\ graphene oxide/carbon nanotubes for as high performance anode for sodium-ion batteries. \\ Electrochimica Acta, 302 65-70.

\section{Authors} \\ Jianmin Feng, Lei Dong, Xifei Li, Dejun Li, Pengyi Lu, Feng Hou, Ji Liang, and Shi Xue Dou
}

This journal article is available at Research Online: https://ro.uow.edu.au/aiimpapers/3486 


\title{
Hierarchically Stacked Reduced Graphene Oxide/Carbon Nanotubes for as High Performance Anode for Sodium-ion Batteries
}

\author{
Jianmin Feng ${ }^{1}$, Lei Dong ${ }^{1}$, Xifei Li $i^{1}$, Dejun $\mathrm{Li}^{1}{ }^{*}$, Pengyi Lu ${ }^{2}$, Feng Hou ${ }^{2}$, Ji Liang ${ }^{3 *}$ \\ and Shi Xue $\mathrm{Dou}^{3}$
}

\begin{abstract}
Author Affiliations:
\end{abstract}
1 Energy and Materials Engineering Center, College of Physics and Materials Science, Tianjin Normal University, No 393 Bin Shui West Road, Xiqing District, Tianjin 300387, P. R. China

2 Key Laboratory of Advanced Ceramics and Machining Technology of Ministry of Education, School of Materials Science and Engineering, Tianjin University, Tianjin 300072, PR China

3 Institute for Superconducting \& Electronic Materials (ISEM), Australian Institute of Innovative Materials (AIIM), Innovation Campus, Squires Way, University of Wollongong, NSW 2522, Australia

*Corresponding Authors: Prof. Dejun Li (dli1961@163.com) and Dr. Ji Liang (liangj@uow.edu.au) 


\begin{abstract}
Sodium has attracted an increasing amount of attention as an alternative element to lithium for energy storage due to its low cost and wide distribution, although the intercalation problem for sodium ions in conventional anode materials, due to their larger ionic size, still has to be overcome in order to utilize this element. Herein, we report a carbon-based hybrid material that is composed of stacked reduced graphene oxide/carbon nanotubes (rGO/CNTs) with a hierarchical and open structure to accommodate Naions, which was fabricated by a liquid-phase oxidative exfoliation and subsequent reduction. This rGO/CNTs hybrid material features a hierarchical nanostructure with rGO nanosheets homogeneously spaced by monodisperse CNTs. The increased interlayer space between individual rGO nanosheets, which resulted from the inserted CNTs, is beneficial for highly efficient and reversible $\mathrm{Na}$ ion intercalation, leading to a high and stable capacity of $295 \mathrm{mAhg}^{-1}$ at $50 \mathrm{mAg}^{-1}$ for 200 cycles.
\end{abstract}




\section{Introduction}

The rapidly growing demand for the various types of renewable energy urgently requires the development of low-cost and durable energy storage strategies; and the electrochemical route, achieved through various batteries or capacitors, has become an ideal option for this purpose, due to its low environmental pollution, high round-trip efficiency, long cycle life, strong applicability, and simple maintenance. ${ }^{[1,2]}$ At this stage, lithium ion batteries (LIBs) are the most popular electrochemical energy storage devices. ${ }^{[3]}$ Unfortunately, with the fast expansion of their applications in various electrical/electronic devices and tools, the gap between the supply and demand of lithium resources has been continuously widened.

In this regard, sodium, the neighboring element to lithium in the alkali metal group, has come into the spotlight as an alternative choice to lithium, due to its much wider distribution and lower cost. ${ }^{[4-6]}$ Sodium shares the same outer orbit electronic structure as lithium and thus exhibits similar chemical properties. In recent studies, it has been proven that a sodium ion battery (SIB) has similar energy storage mechanisms (i.e. by intercalation/de-intercalation and alloying/de-alloying) to a $\mathrm{LIB},{ }^{[7,8]}$ making the SIBs a very promising candidate for next generation energy storage applications, as a low-cost alternative to LIBs.

One significant difference, however, between these two types of batteries, is that $\mathrm{Na}$ ions have a much larger ionic size $(0.102 \mathrm{~nm})$ than that of $\mathrm{Li}$ ions $(0.076 \mathrm{~nm})$, indicating that a larger intercalation/de-intercalation spacing is essential for materials to store $\mathrm{Na}$ ions, and a larger volume variation may occur during the repeated $\mathrm{Na}$ ion insertion and deinsertion. ${ }^{[9,10]}$ In this case, graphite, the commercial anode material for LIBs, is not suitable for $\mathrm{Na}$ ion storage due to its small interlayer distance. ${ }^{[11]}$ Hence, searching for alternative high-performance SIB anode materials has become one of the major tasks in the research on anode materials for SIBs.

With the aim of solving the $\mathrm{Na}$ ion intercalation problem, different kinds of alternative carbon-based materials, which are intrinsically stable in the very harsh battery environment, have been exploited as SIB anode, including hard carbon, ${ }^{[12-15]}$ porous carbons, ${ }^{[16,17]}$ carbon nanotubes, ${ }^{[18-20]}$ graphene, ${ }^{[14,17,18,21-23]}$ and carbon nanofibers. ${ }^{[24]}$ Among them, graphene, the two-dimensional monolayer of $s p^{2}$-hybridized carbon atoms, 
has the most easily accessible structure for $\mathrm{Na}$ ions, in theory. ${ }^{[6,22,23]}$ In practical applications, however, the restacking of graphene nanosheets often occurs and easily blocks the diffusion pathways for $\mathrm{Na}$ ions, making it hard for them to access the inter-sheet space, limiting $\mathrm{Na}$ ion storage capacity, and compromising the reversibility of graphene. ${ }^{[17,25,26]}$

In this work, aimed at these current issues for graphene-based materials and with the purpose of developing a high performance all-carbon SIB anode material, we have designed a reduced graphene oxide/carbon nanotubes (rGO/CNTs) composite material that was fabricated by a straightforward one-step liquid exfoliation technology. In this material, a hierarchical structure has been obtained, with the rGO nanosheets uniformly separated by inserted CNTs, which has effectively prevented the restacking of rGO sheets. Such a hierarchical structure with a stable and large interlayer distance was essential to achieve a high and reversible $\mathrm{Na}$ ion intercalation/deintercalation capacity of $285.6 \mathrm{mAh}$ $\mathrm{g}^{-1}$ at $50 \mathrm{~mA} \mathrm{~g}^{-1}$ after 200 cycles. In addition, this GO/CNTs hybrid material could also be fabricated into a three-dimensional $\mathrm{rGO} / \mathrm{CNT}$ foam for nanostructure optimization, which exhibited an improved $\mathrm{Na}$ ion storage performance of $295 \mathrm{mAh} \mathrm{g}^{-1}$ at $50 \mathrm{~mA} \mathrm{~g}^{-1}$ after 200 cycles.

\section{Experimental}

\subsection{Preparation of $r G O / C N T S$}

Graphite $(0.58 \mathrm{~g})$ was mixed with $\mathrm{KMnO}_{4}(3.48 \mathrm{~g})$ and placed in a three-neck flask. Afterward, $10 \mathrm{ml}$ of phosphoric acid (85\%) and $90 \mathrm{ml}$ of sulfuric acid (98\%) were added into the flask, dropwise and carefully, under continuous stirring in a water bath for $8 \mathrm{~h}$ at $50{ }^{\circ} \mathrm{C}$. Then, $0.5 \mathrm{~g}$ of double-walled CNTs prepared by a chemical vapor process (CVD) [27] and $0.5 \mathrm{~g} \mathrm{KMnO}_{4}$ were added and stirred for another $3 \mathrm{~h}$. A black solution was obtained.

The black solution was poured onto ice $(50 \mathrm{ml})$ in a beaker. Then, $100 \mathrm{ml}$ water and $10 \mathrm{ml}$ of $30 \% \mathrm{H}_{2} \mathrm{O}_{2}$ was dripped in under stirring. Afterward, centrifugation was carried out to separate the GO/CNTs from the solution, and the obtained material was washed with distilled water and ethanol 5 times. Finally, the separated GO/CNTs material was dried at $60{ }^{\circ} \mathrm{C}$. The color of the as-obtained powder was black, which was different from 
that of the yellowish-brown GO.

Then, the dried GO/CNTs sample was reduced through a high temperature annealing process. Specifically, the as-obtained GO/CNTs was ground into fine powder and heated up to $1000{ }^{\circ} \mathrm{C}$ with a ramp rate of $5^{\circ} \mathrm{C} \mathrm{min}^{-1}$ under the protection of Ar. The obtained thermally reduced GO/CNTs sample was denoted as T-rGO/CNTs. For comparison, a $\mathrm{GO} / \mathrm{CNTs}$ sample was also reduced under vacuum at the same temperature (denoted as V-T-rGO/CNTs).

\subsection{Preparation of the foam of $r G O / C N T s$}

Hydrothermal reduction was adopted to prepare the rGO/CNT foam. Specifically, GO/CNTs was dispersed in water by ultrasonication to form an aqueous suspension of

GO/CNTs $\left(4 \mathrm{mg} \mathrm{ml}^{-1}\right.$ ). It was then transferred into a $50 \mathrm{ml}$ Teflon-lined stainless steel autoclave, heated at $180{ }^{\circ} \mathrm{C}$ for $24 \mathrm{~h}$, and cooled down naturally. The obtained hydrogel was freeze-dried to obtain the hydrothermally reduced $\mathrm{rGO} / \mathrm{CNT}$ aerogel foam (denoted as $\mathrm{H}-\mathrm{rGO} / \mathrm{CNT}$ foam). For comparison, $\mathrm{H}-\mathrm{rGO} / \mathrm{CNT}$ foam was further reduced under vacuum at $1000{ }^{\circ} \mathrm{C}$ (with the product denoted as V-H-rGO/CNT foam).

\subsection{Characterizations}

The morphology and structure of the as-prepared products were characterized by scanning electron microscopy (SEM, TDCLS-8010, Hitachi Japan), transmission electron microscopy (TEM, Tecnai G2 F20, FEI, Netherlands), X-ray diffraction (XRD, D8 Advance, Bruker, Germany), and Raman spectroscopy (Horiba Jobin Yvon, LabRAM HR800, $17 \mathrm{~mW}, 514 \mathrm{~nm}$, He-Ne laser). The surface chemistry of rGO/CNTs was analyzed by X-ray photoelectron spectroscopy (XPS, Perkin-Elmer, PHI-1600, US).

\subsection{Electrochemical performance evaluation}

The rGO/CNTs material was mixed with acetylene black powder as the conductive agent and sodium alginate as the binder at a weight ratio of 85:10:5. Then, water was added into the mixture, and it was mechanically ground to form a slurry. The slurry was blade coated on a piece of $\mathrm{Cu}$ foil and dried for $24 \mathrm{~h}$ at $80{ }^{\circ} \mathrm{C}$ under vacuum. The coated foil was then cut into $10 \mathrm{~mm}$-diameter circular disc electrodes.

The electrodes were assembled into coin-type cells (CR2032) in a glove box filled with Ar. $\mathrm{Na}$ foil was used for the counter and reference electrodes. $1 \mathrm{M} \mathrm{NaClO}_{4}$ in ethylene carbonate and dimethyl carbonate (3:7 by volume), and an extra 10\% 
fluoroethylene carbonate were used as electrolyte. A Whatman GF/C borosilicate glass fiber film was used as the separator. Galvanostatic charge-discharge (GCD) tests were performed on a battery testing system (LAND 2001A, Wuhan Jinnuo, China) at a current density of $50 \mathrm{~mA} \mathrm{~g}^{-1}$ within a potential range of $0.01 \mathrm{~V}$ to $3.0 \mathrm{~V}$ vs. $\mathrm{Na}^{+} / \mathrm{Na}$. The cyclic voltammetry $(\mathrm{CV})$ and electrochemical impedance spectroscopy (EIS) investigations on SIBs were conducted on an electrochemical workstation (VersaSTAT4, Princeton Instruments, US).

\section{Results and Discussion}

The fabrication of rGO/CNTs is illustrated in Fig. 1a. Firstly, graphite was treated by Hummer's method to form GO. Afterward, CNTs and $\mathrm{KMnO}_{4}$ were slowly added and further reacted for $24 \mathrm{~h}$ to obtain a homogeneous black solution. After separation and drying, a black product, GO/CNTs, was obtained. Afterward, the rGO/CNTs composite, with rGO nanosheets homogeneously spaced by CNTs, was obtained by reducing the GO/CNTs, either in argon or under vacuum conditions. Such an interspaced nanostructure in the material will be beneficial for facile $\mathrm{Na}$ ion transportation (Fig. 1b).

(a)

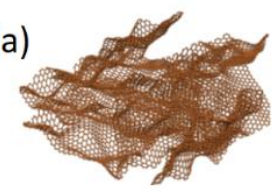

GO

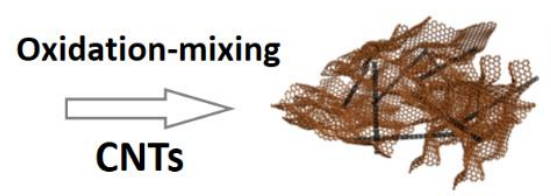

GO/CNTs

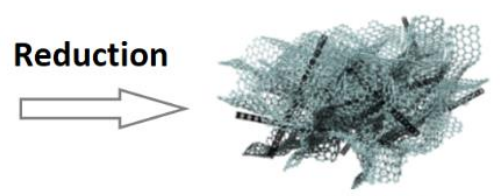

rGO/CNTs

(b)
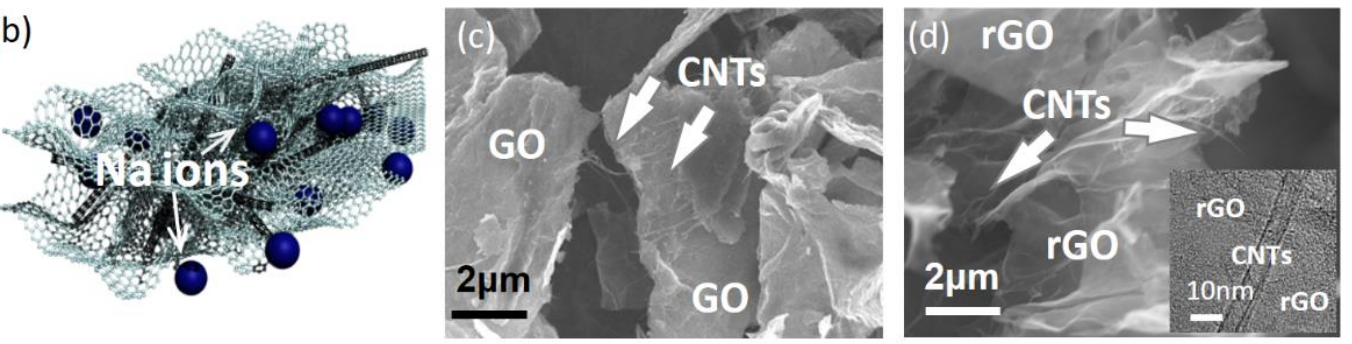

Fig. 1 Schematic illustration of the fabrication of rGO/CNTs (a), schematic illustration of $\mathrm{Na}$ ion transport in rGO/CNTs (b), SEM image of GO/CNTs (c), SEM and TEM (inset) images of T-rGO/CNTs (d).

The morphology of GO/CNTs was first investigated by SEM, which shows a uniform structure consisting of overlapped GO layers and fibrous CNTs (Fig. 1c), clearly showing 
that the CNTs have been uniformly dispersed and exist in between the GO sheets. After the thermal reduction of GO/CNTs, which was carried out at $1000{ }^{\circ} \mathrm{C}$ in $\mathrm{Ar}$, T-rGO/CNTs was obtained, in which the crumbled rGO nanosheets were stacked on each other to form a porous architecture with fibrous CNTs uniformly distributed throughout them (Fig. 1d).From the edges of the rGO layers, extruding CNTs can be clearly seen, further confirming that the rGO sheets have been successfully spaced by the homogeneously dispersed CNTs.

The fine structure of T-rGO/CNTs was further studied using TEM, which showed that the CNTs are accommodated between the rGO layers (inset of Fig. 1d and Fig.2), in agreement with the SEM observations. On the surface of $r G O$, some porous structures could also be found, which were possibly caused by the oxidization upon the addition of KMnO4. ${ }^{[24]}$ In addition, the structure of CNTs have been significantly altered during the oxidization process. Compared with raw CNTs (inset of Fig. 2a), the bundles in the raw CNTs have been dispersed and adhered on the surface of rGO. Meanwhile, the crystallinity of CNTs also deceased and the interlayer distance of the treated CNTs was also enlarged, resulted from the oxidization treatment (inset of Fig. 2b)
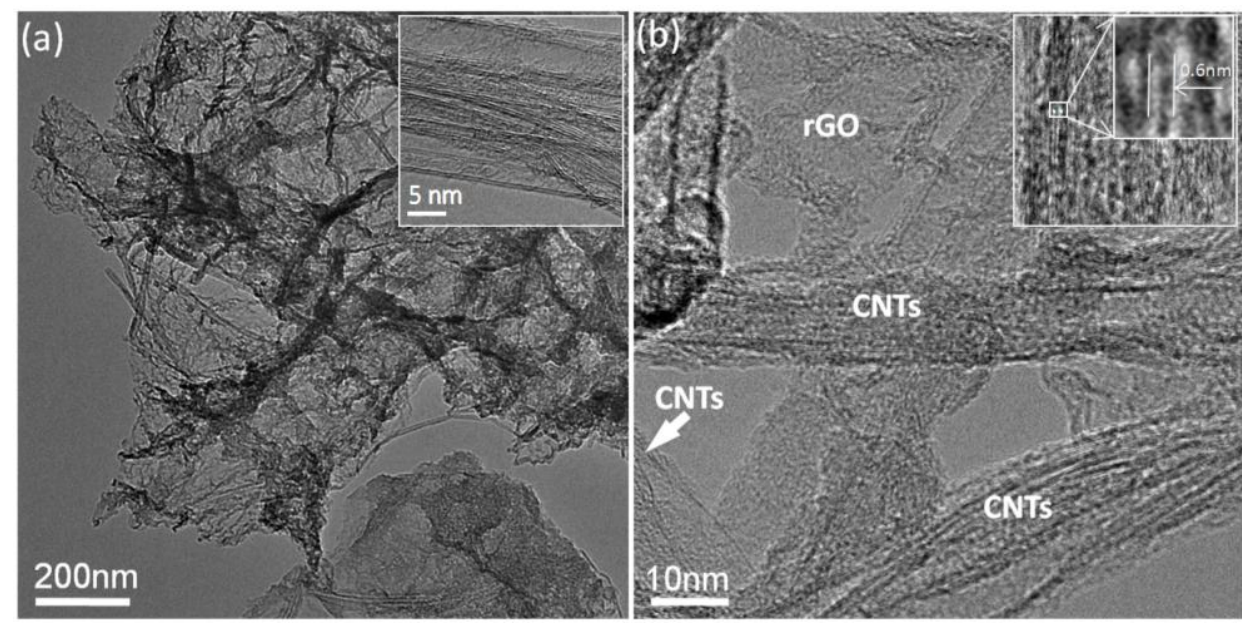

Fig.2 TEM images of T-rGO/CNTS and raw CNTs (inset) (a), HRTEM images of T-rGO/CNTs and enlarged CNT wall (inset) (b). 
Raman analysis was further conducted to reveal more structural information on the T-rGO/CNTs (Fig. 3a). The Raman spectrum of T-rGO/CNTs shows a typical G band at $1590 \mathrm{~cm}^{-1}$ and a D band at $1354 \mathrm{~cm}^{-1}$, with an $I_{\mathrm{D}} / I_{\mathrm{G}}$ intensity ratio of 1.1 . A weak $\mathrm{G}^{\prime}$ band at $2680 \mathrm{~cm}^{-1}$ and D+D' band at $2900 \mathrm{~cm}^{-1}$ could also be observed, which comes from defective graphene layers. ${ }^{[28]}$ Characteristic radial breathing modes (RBMs) of double-walled CNTs can be found at $304 \mathrm{~cm}^{-1} \cdot{ }^{[29]}$ In comparison, in the Raman spectrum of pure rGO, the intensities of the $\mathrm{G}, \mathrm{D}, \mathrm{G}$, and $\mathrm{D}+\mathrm{D}^{\prime}$ bands are lower, and characteristic RBMs of CNTs are also absent. The $I_{\mathrm{D}} / I_{\mathrm{G}}$ ratio of $\mathrm{rGO}$ is 0.78 , which is lower than that of T-rGO/CNTs. It indicates that the T-rGO/CNTs have more defects than rGO, which is in accordance with the TEM observations (Fig. 1d and Fig. 2).
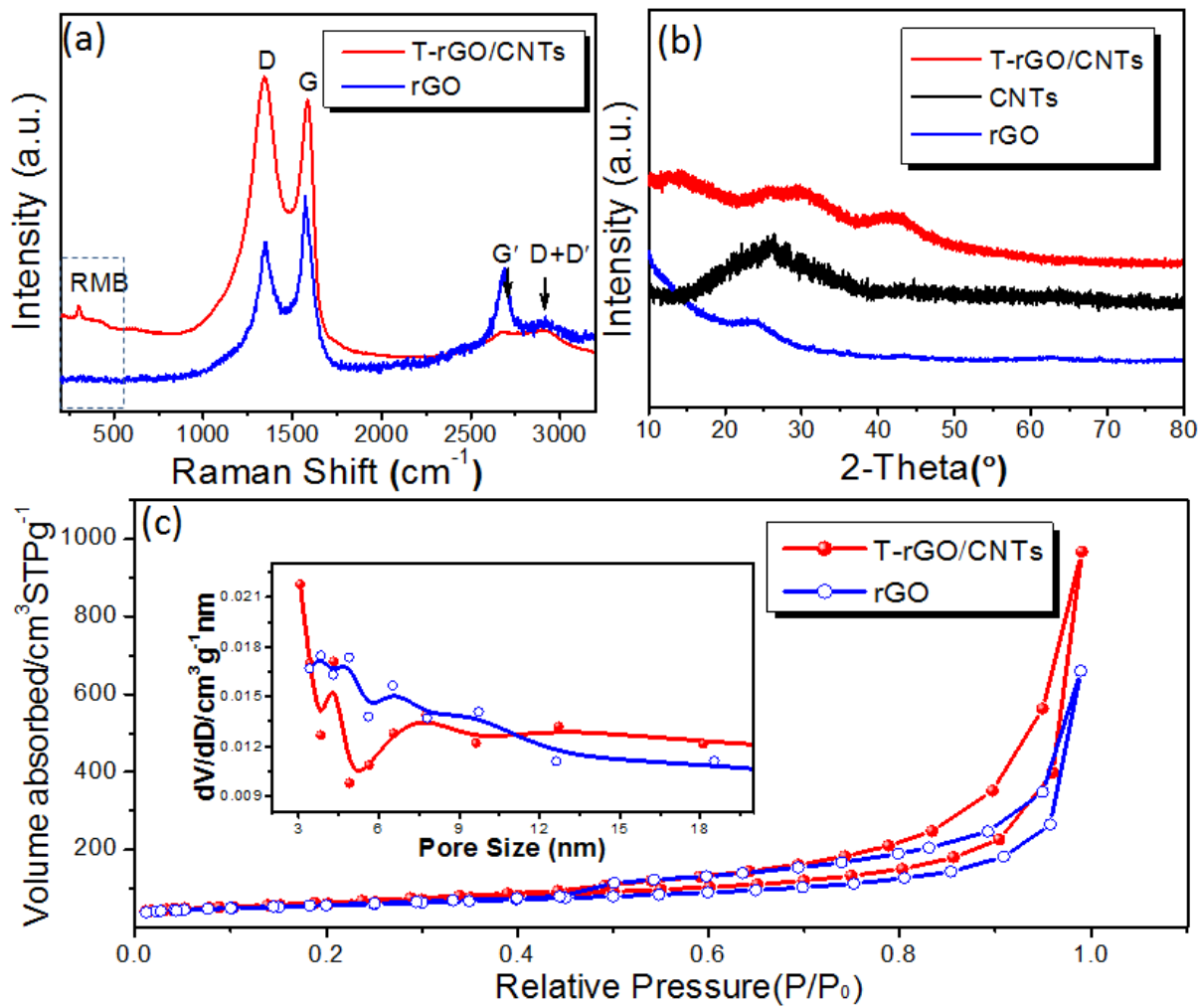

Fig. 3 Raman spectrums of T-rGO/CNTs and rGO (a), XRD patterns of T-rGO/CNTs, CNTs and $r G O(b), N 2$ adsorption-desorption isotherms T-rGO/CNTs and $r G O$.

XRD analysis of T-rGO/CNTs showed that there are three broad peaks at $13.5^{\circ}$, $25^{\circ}-30^{\circ}$, and $41.8^{\circ}$ (Fig. $3 \mathrm{~b}$ ). The ones at $25^{\circ}-30^{\circ}$ and $41.8^{\circ}$ could be ascribed to the 
typical (002) and (100) reflections of the graphitic structure of CNTs; while the one at $13.5^{\circ}$ corresponds to an interlayer distance is $0.65 \mathrm{~nm}$. In contrast, only one poorly defined peak at around $25^{\circ}$ could be seen on the pure rGO, corresponding to a much smaller interlayer distance of only $0.36 \mathrm{~nm}$, due to the restacking of $r G O$ nanosheets without CNTs. For the pure CNTs, only one wide peak at around $25^{\circ}$ could be observed, which is close to the peak position of $r G O$. Hence, it could be speculated that the diffraction peak at $13.5^{\circ}$, which corresponds to the much-enlarged interlayer distance of $0.65 \mathrm{~nm}$, is most likely from the spacing effect of CNTs that prevents the restacking of graphene layers. This significantly increased distance in T-rGO/CNTs composite will be beneficial to improve the kinetics and reversibility of $\mathrm{Na}$ ion intercalation. ${ }^{[11,30]}$

Nitrogen adsorption-desorption isotherms of the materials were then collected to analyse their pore structures (Fig. 3c). An obvious hysteresis loop between desorption and adsorption isotherms can be seen in the medium to high relative pressure region, corresponding to the typical type-IV hysteresis loop. It indicates that the T-rGO/CNTs hybrid has a hierarchical structure with macro- and mesopores. The macropores were probably formed by the curved rGO sheets, while the mesopores originate from the $\mathrm{rGO}$ nanosheets separated by CNTs. This is also in agreement with the pore size distributions of the materials calculated by the Barrett-Joyner-Halenda (BJH) method. Specifically, the pore size of T-rGO/CNTs is centered at $3.81 \mathrm{~nm}$ (inset of Fig. 3c), which is close to the diameter of double-walled CNTs (2-6 nm). In comparison, the pore diameter of rGO is fairly discrete. Moreover, the specific surface area of the material was calculated by the Brunauer-Emmett-Teller (BET) method. The T-rGO/CNTs hybrid has a specific surface area of $263.5 \mathrm{~m}^{2} \mathrm{~g}^{-1}$, which is significantly higher than that of $\mathrm{rGO}\left(32.2 \mathrm{~m}^{2} \mathrm{~g}^{-1}\right)$, further confirming that CNTs have successfully separated rGO nanosheets, thus leading to the exposure of more surface area.

We then evaluated the electrochemical $\mathrm{Na}$ ion storage performance of the materials in a coin cell, with $\mathrm{Na}$ foil as the counter/reference electrode. The $1^{\text {st }}, 10^{\text {th }}, 30^{\text {th }}$, and $50^{\text {th }}$ 
cycle GCD profiles of T-rGO/CNTs, which was tested at $50 \mathrm{~mA} \mathrm{~g}^{-1}$ between 0.01 and 3.0 V vs. Na/ $\mathrm{Na}^{+}$, were collected and are shown in Fig. 4a. The initial discharge and charge capacities were $926 \mathrm{mAh} \mathrm{g}^{-1}$ and $255 \mathrm{mAh} \mathrm{g}^{-1}$, respectively, corresponding to an initial coulombic efficiency of $27.5 \%$. This relatively low initial efficiency might be due to the irreversible reaction of the electrolyte with the surface oxygen-containing groups on $\mathrm{rGO} / \mathrm{CNTs}$ to consume the $\mathrm{Na}$ ions and form a solid electrolyte interphase (SEI). ${ }^{[24,34]} \mathrm{In}$ the subsequent cycles, the material possessed a high and stable Na ion storage capacity of $251 \mathrm{mAh} \mathrm{g}^{-1}$ and coulombic efficiency of nearly $100 \%$, indicating the high reversibility of this material for Na ion storage after the initial SEI formation.
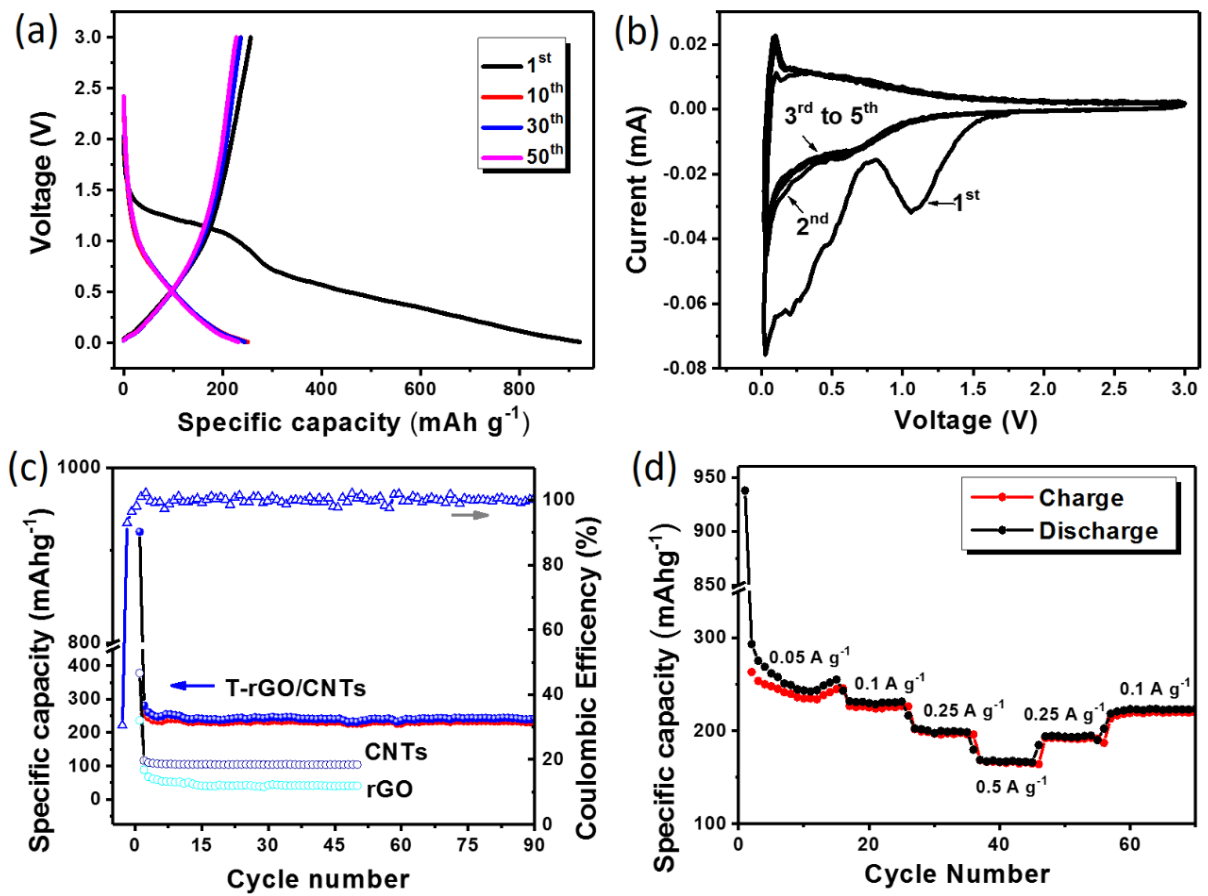

Fig. 4 Charge-discharge curves for T-rGO/CNTs anode at $0.05 \mathrm{~A} \mathrm{~g}^{-1}$ (a), CV curves for the first 5 cycles between 0.01 and $3.0 \mathrm{~V}$ at $0.1 \mathrm{mV} \mathrm{s}^{-1}(\mathrm{~b})$, cycling performance at $0.05 \mathrm{~A}$ $\mathrm{g}^{-1}(\mathrm{c})$, and rate performance at current densities from $0.05 \mathrm{~A} \mathrm{~g}^{-1}$ to $0.5 \mathrm{~A} \mathrm{~g}^{-1}$ (d).

$\mathrm{CV}$ analysis was then conducted to study the $\mathrm{Na}$ ion storage mechanism of this rGO/CNTs material. Fig. $4 \mathrm{~b}$ plots the $\mathrm{CV}$ curves of this $\mathrm{rGO} / \mathrm{CNTs}$ material from the $1^{\text {st }}$ to the $5^{\text {th }}$ cycle. In the negative scan of the $1^{\text {st }}$ cycle, there is one obvious cathodic peak at $1.05 \mathrm{~V}$, corresponding to the SEI formation. It disappears in the subsequent cycles, in 
which only one distinct reduction peak near $0 \mathrm{~V}$ could be observed. On the other hand, in the corresponding positive scan of the first and subsequent cycles, only one small peak at $0.08 \mathrm{~V}$ was observed. After the $1^{\text {st }}$ cycle, the subsequent $\mathrm{CV}$ curves overlap with each other, which indicates stable and reversible $\mathrm{Na}$ ion insertion and deinsertion into/from the material. ${ }^{[24]}$

The cycling performance and rate capability of the T-rGO/CNTs material were then evaluated (Fig. 4c, d). The material shows a stable $\mathrm{Na}$ ion storage performance, with a capacity of $230 \mathrm{mAh} \mathrm{g}^{-1}$ after 90 cycles (Fig. 4c), corresponding to a coulombic efficiency near $100 \%$. In comparison, the Na ion storage capacities of rGO and CNTs are both much lower (40 and $100 \mathrm{mAh} \mathrm{g}^{-1}$, respectively). Hence, these results clearly show that this hybrid structure with rGO nanosheets separated by CNTs could effectively facilitate stable $\mathrm{Na}$ ion storage with high capacity.

The rate performance of $\mathrm{rGO} / \mathrm{CNTs}$ anode was evaluated at various current densities from $0.05 \mathrm{~A} \mathrm{~g}^{-1}$ to $0.5 \mathrm{~A} \mathrm{~g}^{-1}$ (Fig. 4d). At the initial rate of $0.05 \mathrm{~A} \mathrm{~g}^{-1}$, the material possesses a specific capacity of $\sim 250 \mathrm{mAh} \mathrm{g}^{-1}$, which decreases as the current density increases. Even so, at the highest rate of $0.5 \mathrm{~A} \mathrm{~g}^{-1}$, this material still maintains a high capacity of $\sim 168 \mathrm{mAh} \mathrm{g}^{-1}$, and this can be recovered to $190 \mathrm{mAh} \mathrm{g}^{-1}$ at $0.25 \mathrm{~A} \mathrm{~g}^{-1}$ or 230 $\mathrm{mAh} \mathrm{g}^{-1}$ at $0.05 \mathrm{~A} \mathrm{~g}^{-1}$, which is much higher than for the previously reported $\mathrm{rGO}$ and expanded graphite materials. ${ }^{[19]}$

With the advantages of the hierarchical porous structure and enlarged interlayer distance, the T-rGO/CNTs composite exhibits much enhanced performance for $\mathrm{Na}$ ion storage compared with pure rGO or CNTs. ${ }^{[31-33]}$ Unfortunately, the large and irreversible capacity decay in the initial cycle of the T-rGO/CNTs will greatly hinder its practical application. Considering that this issue may be caused by the large amount of residual oxygen-containing groups after the harsh oxidization process, a more complete reduction should be effective in improving the $1^{\text {st }}$ cycle performance of the material.

Thus, a vacuum reduction was carried out by annealing the GO/CNTs under vacuum 
to more effectively remove the oxygen-containing species (i.e. V-T-rGO/CNTs). Under SEM, the homogeneous hybridization of rGO and CNTs could be clearly observed, with CNTs located in between the rGO nanosheets (Fig. 5a, b), which is similar to the case of T-rGO/CNTs. To further confirm the effect of vacuum reduction on the oxygen content of the material, XPS was conducted to compare the surface chemistry of V-T-rGO/CNTs with that of T-rGO/CNTs. It showed that the oxygen content of V-T-rGO/CNTs annealed at $1000{ }^{\circ} \mathrm{C}$ under vacuum was 2.56 at.\%, which was much lower than that of the T-rGO/CNTs (6.59 at.\%, inset of Fig. 5b).
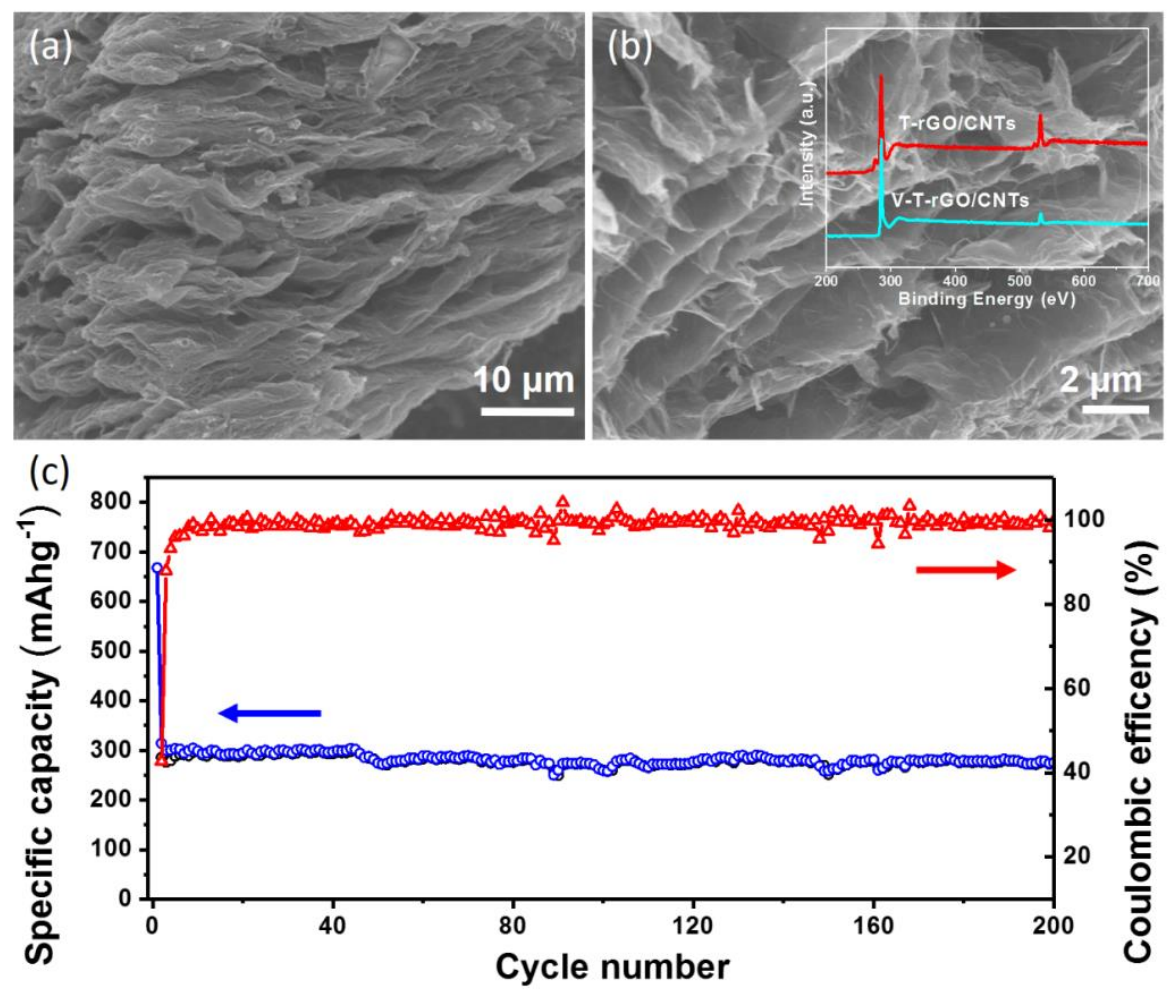

Fig. 5 (a, b) SEM images of V-T-rGO/CNTs, with XPS survey spectra of V-T-rGO/CNTs and T-rGO/CNTs (inset in b); (c) Na ion cycling performance of V-T-rGO/CNTs at $0.05 \mathrm{~A}$ $\mathrm{g}^{-1}$.

The $\mathrm{Na}$ ion storage performance of V-T-rGO/CNTs was then evaluated at $0.05 \mathrm{~mA}$ $\mathrm{g}^{-1}$. The initial discharge and charge capacities of the material are $668.2 \mathrm{mAh} \mathrm{g}^{-1}$ and $285.6 \mathrm{mAh} \mathrm{g}^{-1}$, respectively, corresponding to an initial coulombic efficiency of $42.7 \%$, which is much higher than in the case of T-rGO/CNTs $(27.5 \%)$. It is thus confirmed that 
the vacuum reduction can significantly suppress the undesirable irreversible reactions in the initial cycle and thus effectively improve the $\mathrm{Na}$ ion storage capacity/coulombic efficiency of the material. ${ }^{[34]}$

To better understand the effects of surface oxygen-containing groups on the kinetics of the materials' electrochemical reactions during the insertion/deinsertion of $\mathrm{Na}$ ions, comparative EIS analysis of V-T-rGO/CNTs, T-rGO/CNTs, and rGO was conducted at the charge state after 5 cycles. As shown in Fig. 6a, each Nyquist plot consists of a depressed semicircle in the high to medium frequency region and a straight line in the low frequency region. Based on the Nyquist plots, an equivalent circuit was designed to simulate the electrochemical processes in the materials (Fig. 6b), in which $\mathrm{R}_{\mathrm{s}}, \mathrm{R}_{\mathrm{sf}}, \mathrm{CPE}_{1}$, $\mathrm{R}_{\mathrm{ct}}, \mathrm{CPE}_{2}$, and $\mathrm{W}$ represent the electrolyte resistance, solid electrolyte interphase resistance, surface capacitance, charge transfer resistance, double layer capacitance, and Warburg impedance, respectively. The $\mathrm{R}_{\mathrm{ct}}$ values of $\mathrm{V}-\mathrm{rGO} / \mathrm{CNTs}$, $\mathrm{rGO} / \mathrm{CNTs}$, and rGO are 182.6, 246.5, and $510.2 \mathrm{ohm}$, respectively. Consequently, it could be deduced that the vacuum annealing could not only improve the $\mathrm{Na}$ ion storage efficiency in the initial cycle but also enhance the $\mathrm{Na}$ ion storage kinetics by reducing the internal impedance.
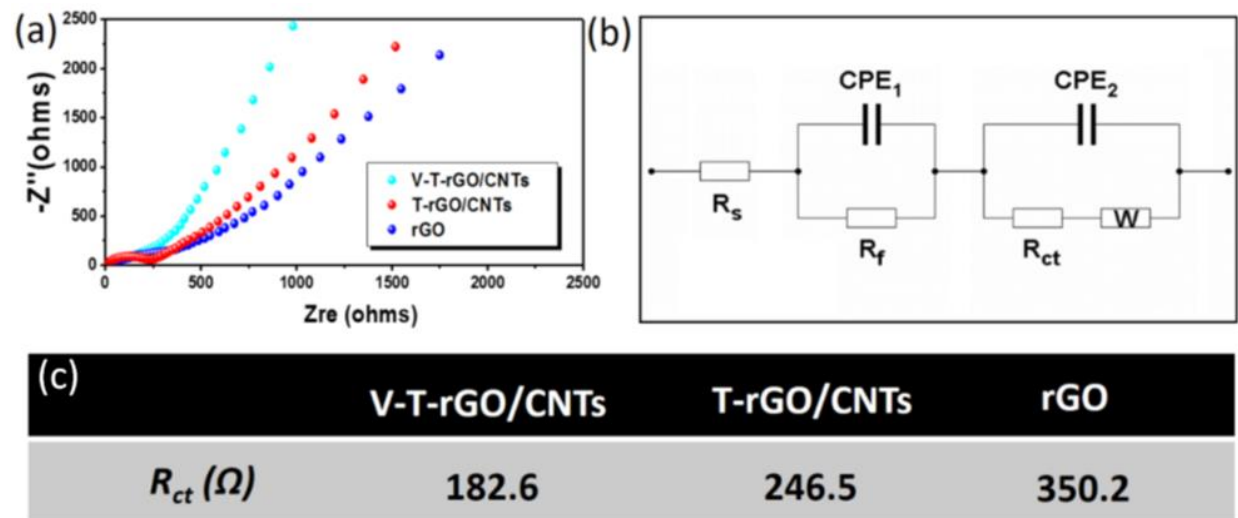

Fig. 6 Nyquist plots of V-rGO/CNTs, T-rGO/CNTs, and rGO reduced in argon (a), fitted equivalent circuit (b), and corresponding charge transfer resistance $R_{c t}$ (c).

In addition, this liquid phase oxidation/exfoliation process, which resulted in a homogeneous liquid suspension of GO and CNTs, also provided the possibility to further 
modify the microstructure through a hydrothermal reduction process, which led to a free-standing and porous rGO/CNTs foam (denoted as H-rGO/CNT foam) (Fig. 7a inset), with rGO sheets forming a three-dimensional porous structure and fibrous CNTs located amongst the rGO nanosheets (Fig. 7a and b).
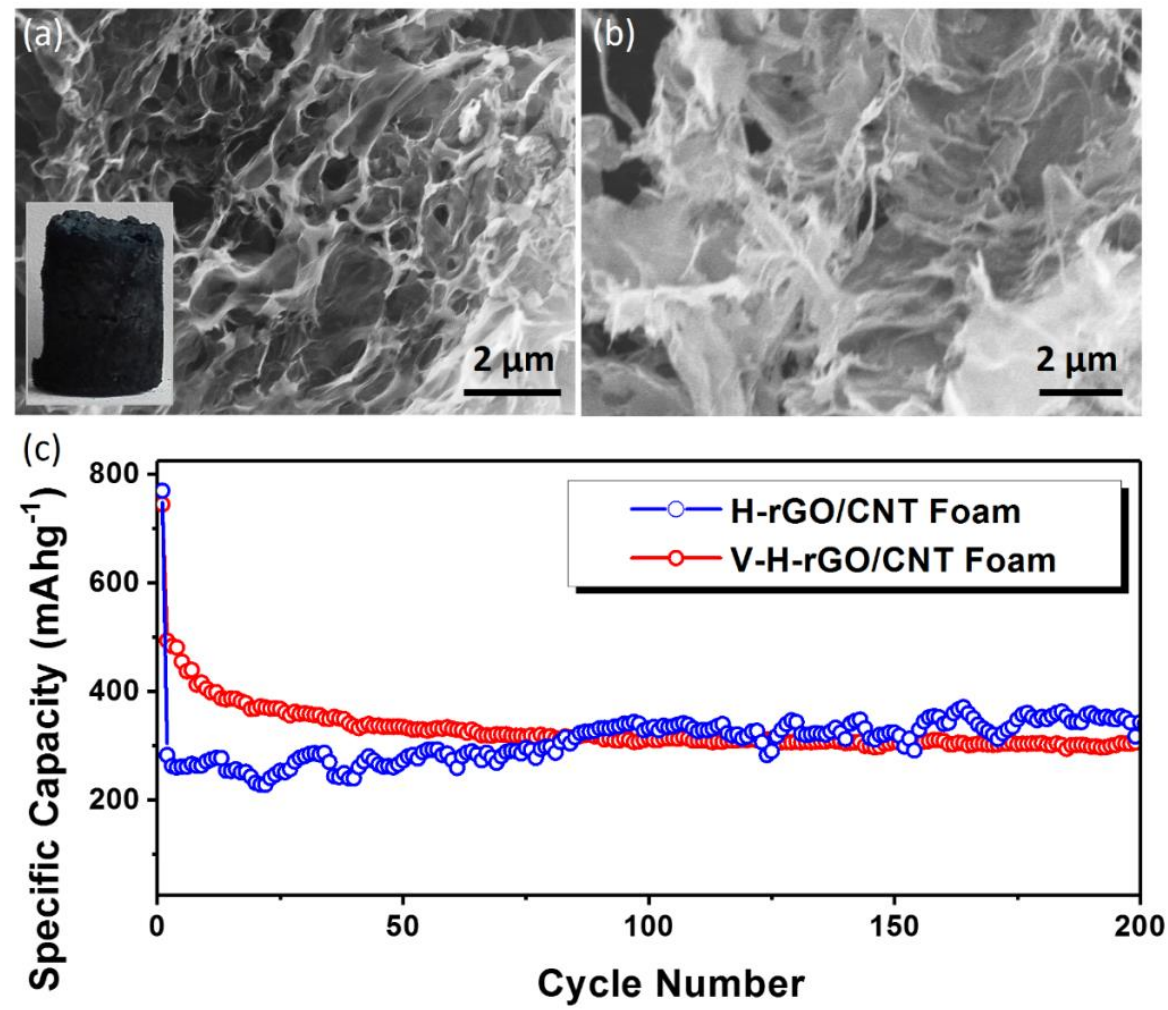

Fig. 7 (a) SEM image of H-rGO/CNT foam and photograph (inset), (b) SEM image of V-H-rGO/CNT foam, (c) SIB anode cycling performance of H-rGO/CNT foam and $\mathrm{V}-\mathrm{H}-\mathrm{rGO} / \mathrm{CNT}$ foam at $0.05 \mathrm{~A} \mathrm{~g}^{-1}$.

The H-rGO/CNT foam was used as SIB anode and tested at a current density of 50 $\mathrm{mA} \mathrm{g}^{-1}$ between 0.01 and $3.0 \mathrm{~V}$ (Fig. 7c). The initial discharge capacity and stable discharge capacity were measured to be $780 \mathrm{mAh} \mathrm{g}^{-1}$ and $\sim 300 \mathrm{mAh} \mathrm{g}^{-1}$, respectively, which was higher than the $285.6 \mathrm{mAhg}^{-1}$ for $\mathrm{V}-\mathrm{H}-\mathrm{rGO} / \mathrm{CNTs}$ in powder form, indicating that this optimized three-dimensional porous structure is beneficial for further improving the electrochemical performance, due to its more facile transport of ions inside its large pores as well as the effective interconnected network to conduct electrons.

Nevertheless, the Na ion storage capacity of $\mathrm{H}-\mathrm{rGO} / \mathrm{CNT}$ foam fluctuated more than 
for $\mathrm{V}-\mathrm{H}-\mathrm{rGO} / \mathrm{CNTs}$ throughout the cycling test, which is possibly due to residual oxygen-containing groups on the surface. Inspired by the improved $\mathrm{Na}$ ion storage performance of V-T-rGO/CNTs via vacuum annealing, the $\mathrm{H}-\mathrm{rGO} / \mathrm{CNT}$ foam was also annealed under vacuum (with the product denoted as V-H-rGO/CNT foam). Compared with the $\mathrm{H}-\mathrm{rGO} / \mathrm{CNT}$ foam, the big pores in $\mathrm{H}-\mathrm{rGO} / \mathrm{CNT}$ foam were hardly observed in $\mathrm{V}-\mathrm{H}-\mathrm{rGO} / \mathrm{CNT}$ foam, although the uniform composite structure of rGO/CNT was maintained. The electrochemical analysis showed that the V-H-rGO/CNT foam had a more stable $\mathrm{Na}$ ion storage capacity of $295 \mathrm{mAh} \mathrm{g}^{-1}$ after 200 cycles. The above analysis further indicates that vacuum reduction annealing could be considered as one of favorable factors for improved $\mathrm{Na}$ ion storage performance of rGO/CNTs.

\section{Conclusions}

In summary, a hybrid nanostructure of $\mathrm{rGO} / \mathrm{CNT}$ s was successfully synthesized via a single liquid-phase oxidization exfoliation process. The unique structure combining two-dimensional graphene and one-dimensional carbon tubes could not only inhibit graphene re-stacking, but could also prevent CNTs from assembling into bundles. Benefiting from the interval effect of the CNTs, the hierarchical porous structure and enlarged interlayer lattice distance promoted the formation of smooth ion channels for $\mathrm{Na}$ ion diffusion. The rGO/CNTs composite had a stable Na ion storage performance of 295 $\mathrm{mAh} \mathrm{g}^{-1}$ at $0.05 \mathrm{~A} \mathrm{~g}^{-1}$ after 200 cycles.

\section{Acknowledgments}

This work is financially supported by the National Natural Science Foundation of China (51772209 and 51472180), the High Technology Research and Development Program of China (863 Program, No. 2015AA034702), the Higher School Science and Technology Development Project of Tianjin City (20140310), the Academic Innovation Fund of Tianjin Normal University (52XC1410), and the Australian Research Council through Discovery Early Career Researcher Award (DECRA, No. DE170100871). We would also like to thank Dr. Tania Silver for the proof reading of the manuscript. 


\section{References}

[1] B. Dunn, H. Kamath, J.-M. Tarascon, Electrical energy storage for the grid: A battery of choices, Science 334(6058) (2011) 928.

[2] D. Kundu, E. Talaie, V. Duffort, L.F. Nazar, The emerging chemistry of sodium ion batteries for electrochemical energy storage, Angew. Chem. Int. Edit. 54(11) (2015) 3431-3448.

[3] L. Wang, Z. Zhou, X. Yan, F. Hou, L. Wen, W. Luo, J. Liang, S.X. Dou, Engineering of lithium-metal anodes towards a safe and stable battery, Energy Storage Mater. 14 (2018) 22-48.

[4] X.-W. Wang, H.-P. Guo, J. Liang, J.-F. Zhang, B. Zhang, J.-Z. Wang, W.-B. Luo, H.-K. Liu, S.-X. Dou, An Integrated Free-Standing Flexible Electrode with Holey-Structured 2D Bimetallic Phosphide Nanosheets for Sodium-Ion Batteries, Adv. Funct. Mater. 28(26) (2018) 1801016.

[5] B. Quan, A. Jin, S.-H. Yu, S.M. Kang, J. Jeong, H.D. Abruña, L. Jin, Y. Piao, Y.-E. Sung, Solvothermal-Derived S-Doped Graphene as an Anode Material for Sodium-Ion Batteries, Adv. Sci. 5(5) (2018) 1700880.

[6] C. Vaalma, D. Buchholz, M. Weil, S. Passerini, A cost and resource analysis of sodium-ion batteries, Nat. Rev. Mater. 3 (2018) 18013.

[7] Y. Li, Y. Lu, C. Zhao, Y.-S. Hu, M.-M. Titirici, H. Li, X. Huang, L. Chen, Recent advances of electrode materials for low-cost sodium-ion batteries towards practical application for grid energy storage, Energy Storage Mater. 7 (2017) 130-151.

[8] M.-S. Balogun, Y. Luo, W. Qiu, P. Liu, Y. Tong, A review of carbon materials and their composites with alloy metals for sodium ion battery anodes, Carbon 98 (2016) $162-178$.

[9] C. Wang, Y. Xu, Y. Fang, M. Zhou, L. Liang, S. Singh, H. Zhao, A. Schober, Y. Lei, Extended $\pi$-conjugated system for fast-charge and -discharge sodium-ion batteries, J. Am. Chem. Soc. 137(8) (2015) 3124-3130.

[10] Y.-J. Kang, S.C. Jung, J.W. Choi, Y.-K. Han, Important role of functional groups for 
sodium ion intercalation in expanded graphite, Chem. Mater. 27(15) (2015) $5402-5406$.

[11] Y. Wen, K. He, Y. Zhu, F. Han, Y. Xu, I. Matsuda, Y. Ishii, J. Cumings, C. Wang, Expanded graphite as superior anode for sodium-ion batteries, Nat. Commun. 5 (2014) 4033.

[12] L. Xiao, Y. Cao, W.A. Henderson, M.L. Sushko, Y. Shao, J. Xiao, W. Wang, M.H. Engelhard, Z. Nie, J. Liu, Hard carbon nanoparticles as high-capacity, high-stability anodic materials for Na-ion batteries, Nano Energy 19 (2016) 279-288.

[13] S. Komaba, W. Murata, T. Ishikawa, N. Yabuuchi, T. Ozeki, T. Nakayama, A. Ogata, K. Gotoh, K. Fujiwara, Electrochemical Na insertion and solid electrolyte interphase for hard-carbon electrodes and application to Na-Ion batteries, Adv. Funct. Mater. 21(20) (2011) 3859-3867.

[14] L. Yin, Y. Wang, C. Han, Y.-M. Kang, X. Ma, H. Xie, M. Wu, Self-assembly of disordered hard carbon/graphene hybrid for sodium-ion batteries, J. Power Sources 305 (2016) 156-160.

[15] C. Bommier, W. Luo, W.-Y. Gao, A. Greaney, S. Ma, X. Ji, Predicting capacity of hard carbon anodes in sodium-ion batteries using porosity measurements, Carbon 76 (2014) 165-174.

[16] H. Hou, C.E. Banks, M. Jing, Y. Zhang, X. Ji, Carbon quantum dots and their derivative 3D porous carbon frameworks for sodium-ion batteries with ultralong cycle life, Adv. Mater. 27(47) (2015) 7861-7866.

[17] Y. Yan, Y.-X. Yin, Y.-G. Guo, L.-J. Wan, A sandwich-like hierarchically porous carbon/graphene composite as a high-performance anode material for sodium-ion batteries, Adv. Energy Mater. 4(8) (2014) 1301584.

[18] X.-F. Luo, C.-H. Yang, Y.-Y. Peng, N.-W. Pu, M.-D. Ger, C.-T. Hsieh, J.-K. Chang, Graphene nanosheets, carbon nanotubes, graphite, and activated carbon as anode materials for sodium-ion batteries, J. Mater. Chem. A 3(19) (2015) 10320-10326. 
[19] S. Licht, A. Douglas, J. Ren, R. Carter, M. Lefler, C.L. Pint, Carbon nanotubes produced from ambient carbon dioxide for environmentally sustainable lithium-ion and sodium-ion battery anodes, ACS Central Sci. 2(3) (2016) 162-168.

[20] H.-g. Wang, Z. Wu, F.-1. Meng, D.-1. Ma, X.-1. Huang, L.-m. Wang, X.-b. Zhang, Nitrogen-doped porous carbon nanosheets as low-cost, high-performance anode material for sodium-ion batteries, ChemSusChem 6(1) (2013) 56-60.

[21] D. Yoon, D.H. Kim, K.Y. Chung, W. Chang, S.M. Kim, J. Kim, Hydrogen-enriched porous carbon nanosheets with high sodium storage capacity, Carbon 98 (2016) 213-220.

[22] A. Ramos, I. Cameán, N. Cuesta, A.B. García, Is single layer graphene a promising anode for sodium-ion batteries?, Electrochim. Acta 178 (2015) 392-397.

[23] D. Datta, J. Li, V.B. Shenoy, Defective graphene as a high-capacity anode material for Na- and Ca-ion batteries, ACS Appl. Mater. Inter. 6(3) (2014) 1788-1795.

[24] L. Fu, K. Tang, K. Song, P.A. van Aken, Y. Yu, J. Maier, Nitrogen doped porous carbon fibres as anode materials for sodium ion batteries with excellent rate performance, Nanoscale 6(3) (2014) 1384-1389.

[25] B. Qu, C. Ma, G. Ji, C. Xu, J. Xu, Y.S. Meng, T. Wang, J.Y. Lee, Layered $\mathrm{SnS}_{2}$-reduced graphene oxide composite-A high-capacity, high-rate, and long-cycle life sodium-ion battery anode material, Adv. Mater. 26(23) (2014) 3854-3859.

[26] L. David, R. Bhandavat, G. Singh, $\mathrm{MoS}_{2}$ /graphene composite paper for sodium-ion battery electrodes, ACS Nano 8(2) (2014) 1759-1770.

[27] J.-M. Feng, R. Wang, Y.-L. Li, X.-H. Zhong, L. Cui, Q.-J. Guo, F. Hou, One-step fabrication of high quality double-walled carbon nanotube thin films by a chemical vapor deposition process, Carbon 48(13) (2010) 3817-3824.

[28] M.S. Dresselhaus, A. Jorio, M. Hofmann, G. Dresselhaus, R. Saito, Perspectives on Carbon Nanotubes and Graphene Raman Spectroscopy, Nano Lett. 10(3) (2010) 751-758. 
[29] S. Bandow, M. Takizawa, K. Hirahara, M. Yudasaka, S. Iijima, Raman scattering study of double-wall carbon nanotubes derived from the chains of fullerenes in single-wall carbon nanotubes, Chem. Phys. Lett. 337(1) (2001) 48-54.

[30] M. Goktas, C. Bolli, E.J. Berg, P. Novák, K. Pollok, F. Langenhorst, M.v. Roeder, O. Lenchuk, D. Mollenhauer, P. Adelhelm, Graphite as cointercalation electrode for sodium - ion batteries: Electrode dynamics and the missing solid electrolyte interphase (SEI), Adv. Energy Mater. 8(16) (2018) 1702724.

[31] X. Jiantie, W. Min, W.N. P., J. Mietek, D. Shixue, D. Liming, High-performance sodium ion batteries based on a $3 \mathrm{~d}$ anode from nitrogen - doped graphene foams, Adv. Mater. 27(12) (2015) 2042-2048.

[32] W. Lv, Z. Li, Y. Deng, Q.-H. Yang, F. Kang, Graphene-based materials for electrochemical energy storage devices: Opportunities and challenges, Energy Storage Mater. 2 (2016) 107-138.

[33] Y. Jiqian, Z. Xianlong, W. Dihua, Z. Xudong, Z. Zhen, S-doped N-rich carbon nanosheets with expanded interlayer distance as anode materials for sodium-ion batteries, Adv. Mater. 29(6) (2017) 1604108.

[34] W. Lei, W. Xiaowei, L.G. Qiang, L.H. Ze, L. Ji, D.S. Xue, Novel surface coating strategies for better battery materials, Surf. Innov. 6(1-2) (2018) 13-18. 